\title{
COVID-19 risk perceptions among healthcare workers during early "new behavior norms" phase
}

Lolita Lolita, Azis Ikhsanudin

Faculty of Pharmacy, Universitas Ahmad Dahlan, Yogyakarta, Indonesia

\begin{tabular}{l} 
Article Info \\
\hline Article history: \\
Received Oct 18, 2021 \\
Revised Nov 24, 2021 \\
Accepted Dec 3, 2021 \\
\hline
\end{tabular}

Keywords:

COVID-19

Crisis control management

Healthcare workers

Indonesia

Perceived risk

\begin{abstract}
The coronavirus disease 2019 (COVID-19) pandemic poses a serious health threat among Indonesian healthcare workers. As front-line workers, they were facing higher risk due to the prolonged exposure of severe acute respiratory syndrome coronavirus 2 (SARS-CoV-2). This study aimed to explore various factors affecting perceived risk among healthcare professionals. A cross-sectional study was conducted among health practitioners who met the inclusion criteria of being over the age of 17 years and resided in Indonesia. This study was conducted during the early "new normal behavior" period from April to July 2020. The sampling technique was convenience sampling which involved collecting data through the online questionnaire. The data were analyzed through the Spearman correlation method using SPSS version 22.0. A total of 248 respondents were included in the final analysis. Overall, age $(\mathrm{p}=0.017)$ and health status $(\mathrm{p}=0.001)$ significantly affected the COVID-19 risk perception among healthcare workers. Therefore, comprehensive COVID-19 risk communication should be established to optimize health behavior and crisis control management among healthcare workers.
\end{abstract}

This is an open access article under the CC BY-SA license.

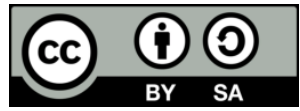

\section{Corresponding Author:}

Lolita Lolita

Faculty of Pharmacy, Universitas Ahmad Dahlan

Prof Dr Soepomo Warungboto Umbulharjo, Yogyakarta, Indonesia

Email: lolita_ur@yahoo.com; lolita@pharm.uad.ac.id

\section{INTRODUCTION}

The novel coronavirus of severe acute respiratory syndrome coronavirus 2 (SARS-CoV-2) causing coronavirus diseases 2019 (COVID-19) has been established as a global health crisis that affected 216 countries of the globe. This virus has rapidly spread to almost all nations, including Indonesia [1]. Indonesia first announced the COVID-19 infection in early March 2020. Within one month, the number of infected people had surpassed nearly 3,000 with an $8 \%$ of case fatality rate. Based on data compiled by Indonesian Health Authorities, there are more than 3.5 million confirmed cases of COVID-19 in the 34 provinces. The significant jump in the number of confirmed COVID-19 positive cases has been followed by a substantial death rate risk [2]. As of June 2020, the Indonesian government presented a "new behavior norms" by liberalized several large scale social restrictions policy to encourage economic, social dan public activities recovery. The implementation of "new behavior norms" provides high public awareness to implement the health protocols when carrying out their normal activities [3].

Many healthcare professionals are at the forefront of an ongoing struggle against the pandemic. They face enormous challenges in providing emergency health care services under extreme pressure [4]. Therefore, they have become at a much greater risk of COVID-19 exposure and might be exposed to significant worsening mental health [5]. A previous study found that depression among health workers was relatively high [6]. Similarly, a study of healthcare workers in Portugal also noted that almost 50\% of them are highly vulnerable to getting COVID-19 infection [7]. Most of them expressed fear of contracting COVID-19 while working in health services. 
According to the Indonesian Medical Association, there has been an increase in the number of COVID-19-related deaths among Indonesian health workers. As of June 2020, 974 health care workers had died as a result of COVID-19; the majority (374 cases) were physicians and nurses (311 cases) [8]. The lack of supply of personal protective equipment (PPE) and inadequate basic training regarding infection control are taking healthcare personnel at greater risk of acquiring the COVID-19 [6]. In addition, dishonest patients in providing healthcare professionals information could be deemed a great challenge in dealing with the pandemic [9]. According to reported cases, many healthcare workers were at high risk of infection during the ongoing COVID-19 pandemic, owing to their close contact with patients and/or potentially infectious co-workers [10].

Risk perception is a major driving factor to elicit individual health behavior in response to the pandemic. Notably, the effectiveness of COVID-19 response strategies will depend upon personal behavior and adherence to COVID-19 preventive measures [11]. Inadequate related knowledge and risk perception among healthcare workers could increase the infection rates caused by delayed therapeutic management of COVID-19 [12]. Understanding the critical factors that influence healthcare workers' risk perception is vital for helping navigate their response in the face of a pandemic [13]. To the best of our knowledge, this is the first study investigating risk perception and its associated factor among Indonesian healthcare workers during the early phase of the new normal-era. Therefore, understanding their COVID-19 risk perception might provide valuable insights to develop more effective risk communications strategies in pandemic prevention and control.

\section{RESEARCH METHOD}

\subsection{Study design and data collection}

This study has been approved by the Research Ethics Committee of Aisyiyah University (reference number: 1305/KEP-UNISA/IV/2020). All participants have been informed about the study purpose and the confidential data detailed on the first page of the online survey. The study was proceeded after obtaining the informed consent and agreement from each participant. A cross-sectional analysis study was designed to examine the risk perception of COVID-19 and its associated factor among Indonesian healthcare professionals. We conducted an online survey questionnaire shared through the social media platforms during the early phase of "new behavior norms" implementation from April to July 2020. The sampling technique was used with non-probability sampling technique, namely convenience sampling. The respondents were identified and chosen from the population based on the relative ease of access to the researchers. The eligibility criteria included healthcare professionals over the age of 17 years and residing in Indonesia. We excluded the participants who did not fill the whole questionnaire and foreign healthcare workers living in Indonesia.

\subsection{Research instrument}

The data collection was carried out by distributing the questionnaire via Google Forms. The particular questionnaire comprised three parts, namely socio-demographic, risk perception, and efficacy belief. The risk perception questionnaire was developed according to our previous study [14], [15]. Before distributing the questionnaire, validity and reliability tests were conducted on 30 anonymous respondents. The content validity test of the questionnaire was performed using the Pearson Product Moment Correlation method with SPSS version 22 . According to the validity test, each item of the questionnaire was valid with a $p$-value $<0.001$. The questionnaire reliability for perceived risk and efficacy belief was 0.806 and 0.734 (Cronbach's alpha). Respondents were asked to answer questionnaires regarding perceived risk dimensions, including perceived severity, vulnerability, followed by two dimensions of efficacy belief, namely response-efficacy and self-efficacy.

Each item questionnaire for perceived severity and perceived vulnerability consisted of one question. We used a 10-point Likert scale to assess the perceived severity of COVID-19, ranging from 1 (not serious) to 10 (very serious). A higher score indicates greater perceived severity of COVID-19. Meanwhile, the perceived vulnerability related to COVID-19 was measured using a five-point Likert scale, ranging from 1 (very unlikely) to 5 (very likely). A higher score indicated a greater exposure of COVID-19 among healthcare workers. The perceived threat was used as the overall measurement of risk perception, calculated using the square root of the multiplication of perceived severity divided by two and perceived vulnerability. The result was a perceived threat using a five-point Likert scale ranging from 1 (low) to 5 (high). The response-efficacy was assessed by asking how confident the participants think the people around them can take practical actions to prevent contracting COVID-19 using a 4-point Likert scale from 1 (not at all) to 4 (very much). Furthermore, selfefficacy was assessed by asking how confident they think they can prevent contracting the disease. The answer options used a 4-point Likert scale, from 1 (not confident) to 4 (very confident) [14], [15].

\subsection{Data analysis}

All statistical software packages were conducted using SPSS version 22.0 for data entry and analysis (IBM Corp., Armonk, NY, USA). Descriptive analysis was carried out to describe the sociodemographic characteristics as independent variables such as sex, age, educational level, marital status, 
health status, quarantine condition, chronic illness history, smoking history, supplement use, and availability of personal protective equipment (PPE) during the pandemic. In comparison, the dependent variable was risk perception of COVID-19 in three categories: perceived severity, perceived vulnerability, and perceived threat. The data was tested using the Kolmogorov-Smirnov to examine the normal distribution between variables. The Kruskal-Wallis/Mann Whitney $U$ bivariate analysis was performed to determine the relationship between sociodemographic characteristics towards risk perception dimensions and efficacy beliefs. The Spearman correlation test was carried out to identify the association of sociodemographic factors with risk perception of COVID-19. A significance level of 5\% was considered statistically significant.

\section{RESULTS AND DISCUSSION}

In total, 251 participants have completed filling and submitting the questionnaire. Furthermore, data were checked to ensure that the whole respondents met the inclusion and exclusion criteria. Three data were excluded because the respondents did not fill the whole questionnaire. A total of 248 participants were input into the database and used for data analysis.

\subsection{Demographic}

Table 1 describes that majority of respondents were female (75.8\%), adult (72.6\%), and had a higher education (96.8\%). Most of the participants in this study were health workers who had a healthy condition $(83.9 \%)$, consumed supplements routinely $(82.3 \%)$, and still worked in health services every day $(58.5 \%)$.

Table 1. Demographic characteristic of healthcare workers

\begin{tabular}{|c|c|c|}
\hline Variables & $\mathrm{n}$ & $\%$ \\
\hline \multicolumn{3}{|l|}{ Gender } \\
\hline Male & 60 & 24.2 \\
\hline Female & 188 & 75.8 \\
\hline \multicolumn{3}{|l|}{ Age } \\
\hline Adolescent & 46 & 18.5 \\
\hline Adult & 180 & 72.6 \\
\hline Elderly & 22 & 8.9 \\
\hline \multicolumn{3}{|l|}{ Education } \\
\hline Middle education & 8 & 3.2 \\
\hline Higher education & 240 & 96.8 \\
\hline \multicolumn{3}{|l|}{ Marital status } \\
\hline Married & 173 & 69.8 \\
\hline Single & 68 & 27.4 \\
\hline Widow/Widower & 7 & 2.8 \\
\hline \multicolumn{3}{|l|}{ Health status } \\
\hline Healthy & 208 & 83.9 \\
\hline Do not know & 18 & 7.3 \\
\hline Doubtful & 7 & 2.8 \\
\hline Probable & 2 & 0.8 \\
\hline Suspect & 10 & 4.0 \\
\hline It has been declared cured of COVID-19 & 3 & 1.2 \\
\hline \multicolumn{3}{|l|}{ Quarantine conditions } \\
\hline Full time activities at home & 21 & 8.5 \\
\hline Still leaving the house $2-3 \mathrm{x}$ a week is not for work & 10 & 4.0 \\
\hline Work outside the home every day & 145 & 58.5 \\
\hline Work outside the home $2-3 \mathrm{x}$ a week & 56 & 22.6 \\
\hline Others & 16 & 6.5 \\
\hline \multicolumn{3}{|l|}{ History of chronic illness } \\
\hline Yes & 15 & 6 \\
\hline No & 233 & 94 \\
\hline \multicolumn{3}{|l|}{ Smoking history } \\
\hline Yes & 17 & 6.9 \\
\hline No & 231 & 93.1 \\
\hline \multicolumn{3}{|l|}{ Supplements use } \\
\hline Yes & 204 & 82.3 \\
\hline No & 44 & 17.7 \\
\hline \multicolumn{3}{|l|}{ Personal protective equipment (PPE) use } \\
\hline Yes & 143 & 57.7 \\
\hline Sometimes & 74 & 29.8 \\
\hline Rarely & 18 & 7.3 \\
\hline Never & 12 & 4.8 \\
\hline
\end{tabular}

Int J Public Health Sci, Vol. 11, No. 1, March 2022: 352-358 


\subsection{Healthcare professional's risk perception}

Table 2 reveals the sociodemographic differences towards perceived risk and efficacy belief. Our study found that several sociodemographics have significant differences with risk perception and efficacy belief, including gender, age, marital status, health status, quarantine condition, and use of PPE. Regarding the sex group, this variable was significantly different from the perceived severity domain $(\mathrm{p}=0.001)$. The average value of perceived severity among women healthcare workers was significantly higher than that of men. This finding indicated that women were aware of the COVID-19 severity while working as health care providers during the pandemic. They also believed that COVID-19 might pose a risk of contracting the more severe disease. A study conducted by Sriharan et al. found that women healthcare workers frequently experienced emotional and mental health problems, contributing to their fear of contracting COVID-19 [16]. In addition, psychosocial and behavioral factors also played a role in gender differences in the perceived pandemic severity. According to another study, men were more likely to underestimate the severity of the potential virus that could harm them [17]. Meanwhile, women reported avoiding large public gatherings or closed physical contact with other people [18].

Our study also found a significant difference in perceived vulnerability $(\mathrm{p}=0.027)$ and response efficacy $(\mathrm{p}=0.034)$ according to marital status. Widow or widower health workers perceived themselves as more vulnerable to COVID-19 compared to married or single workers. This result indicated that widow or widower health workers believe their chances of contracting COVID-19 are incredibly high, leading them to take preventive measurements. A previous study also concluded that a high level of perceived vulnerability among health care workers was triggered by workload factors and a lack of social support from closest friends or family [16].

These findings also revealed that health status had a significant difference with perceived vulnerability. Healthcare workers who perceived themselves as in good health during the COVID-19 pandemic had a lower perceived vulnerability than other groups. According to existing data, individuals with pre-existing medical conditions are more susceptible to death [19], [20].

The existence of a quarantine policy might affect health workers' perceptions during the COVID-19 pandemic. In this study, there was a significant difference between quarantine conditions and perceived severity $(\mathrm{p}=0.016)$. The perceived severity of COVID-19 was lower among healthcare workers who left the house 2-3 times per week for non-work reasons, implying that they did not consider COVID-19 as a severe disease. During the COVID-19 pandemic, quarantine had a substantial impact on stress levels, especially for healthcare personnel. Those who continue to work during quarantine are seen as more vulnerable than those who do not [21].

There were significant differences in PPE use towards response-efficacy $(\mathrm{p}=0.023)$ and self-efficacy $(p=0.017)$. The self-efficacy and response efficacy of health workers who did not wear PPE were lower than others, suggesting that they might not be able to face the threat of COVID-19 and not take COVID-19 precautions. A study supported these findings, which reported that adequate information about the use of PPE influences the risk perception towards infection. As frontline healthcare workers fighting this pandemic, they should know how to use PPE to prevent SAR-CoV-2 exposure properly [22]. Our findings also highlighted the consistent availability of PPE and its supply for healthcare workers. According to our study, more than half of the participants (66.1\%) stated that PPE availability was at an adequate stock level. Optimization of the PPE supply chain is crucial in enabling safe and effective infection prevention of COVID-19 disease.

Individuals' perceived risks constitute a significant component in health behavior and risk communication, reflecting the individuals' judgment about their potential harm such as injury, illness, and death. Risk perception may be related to several factors of individual sociodemographic. According to Table 3 , it could be seen that age ( $p$-value $=0.017)$, marital status ( $p$-value $=0.031$ ), and health status ( $p$-value $=0.001$ ) have a significant correlation towards healthcare workers' risk perception.

Spearman correlation analysis indicated a significant negative correlation between age and risk perception $(\mathrm{r}=-0.152, \mathrm{p}$-value $=0.017)$. It implies that the higher of healthcare worker's age, the lower their COVID-19 risk perception. The present findings suggested that elderly healthcare workers are less perceived regarding the COVID-19 fatality risk. In line with several studies stated that older adults were associated with a lower risk for contracting COVID-19 and less experiencing negative emotions, including anxiety and depression [23]-[25]. Older people are more resilient with minor complaints in daily life in order to avoid becoming stressed. Although the elderly reported fewer potentially stressful events, they described them as less unpleasant [26]. Another study concluded that younger age has a greater COVID-19 threat concerns while older adults had better emotional well-being and were less reactive to stressors in the early weeks of the pandemic [27]. Despite the COVID-19 epidemic, older adults might have managed their emotions by focusing on positive activities and interactions to reduce their stress [28].

According to this research, a significant positive relationship has been reported between risk perception and health status. These findings demonstrated that healthcare workers perceived as at high risk of contracting COVID-19 would take proactive preventive measures to avoid infection. As previously reported, the higher risk perception among healthcare workers contributes to them adhering to health protocols and engaging in the preventive health behaviors related to COVID-19 [11]. 
Table 2. Healthcare workers perceived risk towards COVID-19



Risk perception has long been recognized as a critical concept in encouraging healthy behaviors. Managing public health risks during the COVID-19 pandemic depended on the public's ability to assess their potential risks [29]. COVID-19 pandemic awareness was processed according to each individual's risk 
perception [30]. Therefore, it is important to understand the impact of numerous factors on COVID-19 risk perception. A high level of risk perception among healthcare workers will increase their awareness in implementing protective behaviors to contribute significantly to COVID-19 prevention and control [31].

Table 3. Factor correlation analysis of healthcare risk perception towards COVID-19

\begin{tabular}{lcc}
\hline \multirow{2}{*}{ Independent variables } & \multicolumn{2}{c}{ Risk perception } \\
& Coefficient correlation & p-value \\
\hline Age & -0.152 & $0.017^{*}$ \\
Education & -0.034 & 0.592 \\
Health status & 0.205 & $0.001^{*}$ \\
Quarantine conditions & 0.104 & 0.102 \\
The use of PPE & -0.037 & 0.559 \\
\hline
\end{tabular}

\section{CONCLUSION}

Healthcare workers are at disproportionate risk of contracting COVID-19. Evaluating the risk perception and its associated factors is vital in identifying healthcare workers' protective action in handling the pandemic. This study identified healthcare workers with a high level of COVID-19 risk perception during the early phase of new normal behavior. Age and health status are significantly correlated with the COVID-19 risk perception. Therefore, comprehensive risk communication, professional training, and great support should be implemented to strengthen the pandemic management strategies in the healthcare workforce setting.

This study has several limitations, including the sample being less representative of the Indonesian healthcare workers population. It is more likely that better results will be obtained from a larger sample size. Moreover, the cross-sectional study methodology could only illustrate relationships between patterns and social-demographic factors; no causal relationship exists. A further cohort-based study design should be proposed to measure changes in COVID-19 risk perception across time. Therefore, this study should be considered a preliminary study as such, and the findings can be used to help improve risk communication and epidemic control education in the future pandemic.

Despite these limitations, our present findings might be expected to enhance risk perception, promote preventive actions, and initiate online sessions to assist healthcare professionals in comprehending existing standards and protecting themselves from COVID-19 infection. Targeted risk communication with relevant channels should be considered for these frontline groups to improve their risk awareness and safety actions.

\section{REFERENCES}

[1] Z. Xu et al., "Pathological findings of COVID-19 associated with acute respiratory distress syndrome," The Lancet. Respiratory medicine, vol. 8, no. 4. pp. 420-422, Apr. 2020, doi: 10.1016/S2213-2600(20)30076-X.

[2] WHO Indonesia, "Coronavirus disease 2019 (COVID-19) situation report-18," World Heal. Organ, 2020.

[3] H. Andriani, S.Si, Apt, M.Sc, Ph.D, "Effectiveness of large-scale social restrictions (PSBB) toward the New Normal Era during COVID-19 outbreak: a mini policy review," J. Indones. Heal. Policy Adm., vol. 5, no. 2, pp. 61-65, 2020, doi: 10.7454/ihpa.v5i2.4001.

[4] Q. Liu et al., "The experiences of health-care providers during the COVID-19 crisis in China: a qualitative study," Lancet Glob. Heal., vol. 8, no. 6, pp. e790-e798, 2020, doi: 10.1016/S2214-109X(20)30204-7.

[5] L. Kang et al., "The mental health of medical workers in Wuhan, China dealing with the 2019 novel coronavirus.," The lancet. Psychiatry, vol. 7, no. 3. p. e14, Mar. 2020, doi: 10.1016/S2215-0366(20)30047-X.

[6] S. C. Lam et al., "Perceived risk and protection from infection and depressive symptoms among healthcare workers in Mainland China and Hong Kong During COVID-19," Frontiers in Psychiatry, vol. 11. p. 686, 2020.

[7] D. Peres, J. Monteiro, M. Almeida, and R. Ladeira, "Risk perception of COVID-19 among the portuguese healthcare professionals and general population," J. Hosp. Infect., vol. 105, no. 3, pp. 434-437, May 2020, doi: 10.1016/j.jhin.2020.05.038.

[8] WHO Indonesia, "Coronavirus disease 2019 (COVID-19) situation report-60," World Heal. Organ, 2020.

[9] Y. Zolkefli, "Be honest: individuals' moral responsibility within the COVID-19 context," Malaysian J. Med. Sci., vol. 27, no. 6, pp. 144-147, 2020, doi: 10.21315/mjms2020.27.6.13.

[10] A. Soebandrio et al., "COVID-19 prevalence among healthcare workers in Jakarta and neighbouring areas in Indonesia during early 2020 pandemic,” Ann. Med., vol. 53, no. 1, pp. 1896-1904, Jan. 2021, doi: 10.1080/07853890.2021.1975309.

[11] S. Girma, L. Agenagnew, G. Beressa, Y. Tesfaye, and A. Alenko, "Risk perception and precautionary health behavior toward COVID-19 among health professionals working in selected public university hospitals in Ethiopia," PLoS One, vol. 15, no. 10, p. e0241101, 2020, doi: 10.1371/journal.pone.0241101.

[12] A. S. Bhagavathula, W. A. Aldhaleei, J. Rahmani, M. A. Mahabadi, and D. K. Bandari, "Knowledge and Perceptions of COVID-19 Among Health Care Workers: Cross-Sectional Study," JMIR Public Heal. Surveill., vol. 6, no. 2, p. e19160, Apr. 2020, doi: 10.2196/19160.

[13] J. Jacobs, M. Taylor, K. Agho, G. Stevens, M. Barr, and B. Raphael, "Factors associated with increased risk perception of pandemic influenza in australia," Influenza Res. Treat., vol. 2010, p. 947906, 2010, doi: 10.1155/2010/947906.

[14] R. O. Nanda et al., "COVID-19 risk perception among Indonesians in early stage of the outbreak," Int. J. Public Heal. Sci., vol. 10, no. 2, pp. 249-257, 2021, doi: 10.11591/ijphs.v10i2.20678.

[15] R. O. Nanda, L. Lolita, W. Indayati, I. Rusdiyanti, A. Ikhsanudin, and S. Mareti, "Knowledge, precautionary actions, and perceived risk of COVID-19 among indonesian people," International Journal Public Health Science (IJPHS), vol. 10, no. 1, pp. 
8-15, 2021, doi: 10.11591/ijphs.v10i1.20589.

[16] A. Sriharan et al., "Occupational stress, burnout, and depression in women in healthcare during COVID-19 Pandemic: rapid scoping review," Front. Glob. Women's Heal., vol. 1, no. November, pp. 1-8, 2020, doi: 10.3389/fgwh.2020.596690.

[17] G. Sharma, A. S. Volgman, and E. D. Michos, "Sex differences in mortality from COVID-19 pandemic: are men vulnerable and women protected?," JACC. Case reports, vol. 2, no. 9, pp. 1407-1410, Jul. 2020, doi: 10.1016/j.jaccas.2020.04.027.

[18] D. M. Griffith et al., "Men and COVID-19: a biopsychosocial approach to understanding sex differences in mortality and recommendations for practice and policy interventions," Prev. Chronic Dis., vol. 17, p. E63, Jul. 2020, doi: $10.5888 / \mathrm{pcd} 17.200247$.

[19] G. Giangreco, "Case fatality rate analysis of Italian COVID-19 outbreak," J. Med. Virol., vol. 92, no. 7, pp. 919-923, Jul. 2020, doi: $10.1002 / \mathrm{jmv} .25894$

[20] C. Niepel, D. Kranz, F. Borgonovi, V. Emslander, and S. Greiff, "The coronavirus (COVID-19) fatality risk perception of US adult residents in March and April 2020," Br. J. Health Psychol., vol. 25, no. 4, pp. 883-888, Nov. 2020, doi: 10.1111/bjhp.12438.

[21] J. Torales et al., "Self-perceived stress during the quarantine of COVID-19 Pandemic in paraguay: an exploratory survey," Front. psychiatry, vol. 11, p. 558691, 2020, doi: 10.3389/fpsyt.2020.558691.

[22] E. Savoia, G. Argentini, D. Gori, E. Neri, R. Piltch-Loeb, and M. P. Fantini, "Factors associated with access and use of PPE during COVID-19: A cross-sectional study of Italian physicians," PLoS One, vol. 15, no. 10, p. e0239024, 2020, doi: 10.1371/journal.pone.0239024.

[23] W. Bruine de Bruin, "Age differences in COVID-19 risk perceptions and mental health: evidence from a national U.S. survey conducted in March 2020," J. Gerontol. B. Psychol. Sci. Soc. Sci., vol. 76, no. 2, pp. e24-e29, Jan. 2021, doi: 10.1093/geronb/gbaa074.

[24] L. L. Carstensen, M. Pasupathi, U. Mayr, and J. R. Nesselroade, "Emotional experience in everyday life across the adult life span.," J. Pers. Soc. Psychol., vol. 79, no. 4, pp. 644-655, Oct. 2000.

[25] B. Löwe et al., "A 4-item measure of depression and anxiety: validation and standardization of the Patient Health Questionnaire-4 (PHQ-4) in the general population," J. Affect. Disord., vol. 122, no. 1-2, pp. 86-95, Apr. 2010, doi: 10.1016/j.jad.2009.06.019.

[26] A. B. Neubauer, J. M. Smyth, and M. J. Sliwinski, "Age differences in proactive coping with minor hassles in daily life," $J$. Gerontol. B. Psychol. Sci. Soc. Sci., vol. 74, no. 1, pp. 7-16, Jan. 2019, doi: 10.1093/geronb/gby061.

[27] P. Klaiber, J. H. Wen, A. DeLongis, and N. L. Sin, "The ups and downs of daily life during COVID-19: age differences in affect, stress, and positive events," Journals Gerontol. Ser. B, vol. 76, no. 2, pp. e30-e37, Feb. 2021, doi: 10.1093/geronb/gbaa096.

[28] L. L. Carstensen, "The influence of a sense of time on human development," Science, vol. 312, no. 5782, pp. 1913-1915, Jun. 2006, doi: 10.1126/science. 1127488 .

[29] T. Wise, T. D. Zbozinek, G. Michelini, C. C. Hagan, and D. Mobbs, "Changes in risk perception and self-reported protective behaviour during the first week of the COVID-19 pandemic in the United States," R. Soc. open Sci., vol. 7, no. 9, p. 200742, Sep. 2020, doi: $10.1098 /$ rsos.200742

[30] L. Simione and C. Gnagnarella, "Differences between health workers and general population in risk perception, behaviors, and psychological distress related to COVID-19 spread in Italy," Frontiers in Psychology, vol. 11. p. 2166, 2020.

[31] A. Gorini et al., "Mental health and risk perception among Italian healthcare workers during the second month of the COVID-19 pandemic," Arch. Psychiatr. Nurs., vol. 34, no. 6, pp. 537-544, Dec. 2020, doi: 10.1016/j.apnu.2020.10.007.

\section{BIOGRAPHIES OF AUTHORS}

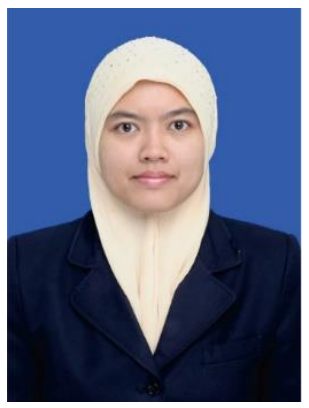

Lolita (iD 8. SC P is an Assistant Professor of Universitas Ahmad Dahlan, Yogyakarta, Indonesia. She has published some scientific articles related to pharmacogenetic, social pharmacy, community health at International Journal which indexed in scopus and web of science. Her research interest in the topic of pharmacy and community health including clinical pharmacy, pharmacogenetics, and epidemiology. She is also a reviewer in scopus indexed international journals. She can be contacted at email: lolita_ur@yahoo.com; lolita@pharm.uad.ac.id.

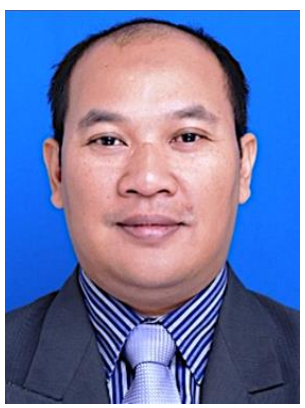

Azis Iksanudin (D) SC P is an Assistant professor in Faculty of Pharmacy, Universitas Ahmad Dahlan, Yogyakarta Indonesia. His research interests are in the field of pharmacy including pharmaceutical technology, natural product pharmacology, biomaterial, and drug delivery. He has many experience in conduct a researched granted from Higher Education of Indonesia and published some articles in Indonesian-accredited journal and scopus-indexed international journals. He can be contacted at email: azis.ikhsanudin@ pharm.uad.ac.id. 